\title{
GMR
}

\section{Differential expression of hENT1 and hENT2 in colon cancer cell lines}

\author{
Y. Liu ${ }^{1}$, T. Zuo ${ }^{1}$, X. Zhu ${ }^{1}$, N. Ahuja ${ }^{2}$ and T. Fu ${ }^{1}$ \\ ${ }^{1}$ Department of Gastrointestinal Surgery II, Key Laboratory of Hubei \\ Province for Digestive System Disease, Renmin Hospital, Wuhan University, \\ Wuhan, China \\ ${ }^{2}$ Department of Surgery, Oncology, Urology, \\ The Johns Hopkins University School of Medicine, Baltimore, MD, USA \\ Corresponding author: T. Fu \\ E-mail: futao1975@tom.com \\ Genet. Mol. Res. 16 (1): gmr16019549 \\ Received November 22, 2016 \\ Accepted December 23, 2016 \\ Published February 16, 2017 \\ DOI http://dx.doi.org/10.4238/gmr16019549 \\ Copyright $(2017$ The Authors. This is an open-access article distributed under the terms of \\ the Creative Commons Attribution ShareAlike (CC BY-SA) 4.0 License.
}

\begin{abstract}
Human equilibrative nucleoside transporters (hENT) 1 and 2, encoded by SLC29A1 and SLC29A2, permit the bidirectional passage of nucleoside analogues into cells and may correlate with clinical responses to chemotherapy in patients with colorectal cancer (CRC). The purpose of this study was to evaluate the expression profiles of SLC29A1 and SLC29A2 in human cancer cell lines. Using quantitative real-time polymerase chain reaction, we comprehensively profiled the transcription levels of SLC29A1 and SLC29A2 in 16 colon cancer cell lines. We validated the ubiquitous and heterogeneous distribution of SLC29A1 and SLC29A2 in human colon cancer cell lines and demonstrated that SLC29A1 was highly expressed in $25 \%$ of metastatic cell lines (Colo201 and Colo205) and 62.5\% of primary cell lines (Caco2, Colo320, HCT116, RKO, and SW48). For the first time, we showed that both $S L C 29 A 1$ and SLC29A2 were expressed at lower levels in colon cancer cell lines originating from metastatic sites than from primary sites. These findings indicate that most patients with metastatic
\end{abstract}

Genetics and Molecular Research 16 (1): gmr16019549 
CRC (mCRC) may have low hENT1 expression, and treatment with nucleoside analogues may be inefficient. However, some patients still show high hENT1 expression and have a high probability of benefiting from these drugs. Therefore, evaluating transporter expression profiles and different drug responses between primary and metastatic tumors in patients with mCRC is important. Further assessment of the association between hENTs and drug-based treatment of mCRC is required to elucidate the mechanisms of chemotherapy resistance.

Key words: hENT1; hENT2; Colon cancer; Expression; Polymerase chain reaction

\section{INTRODUCTION}

Human genome sequence analysis indicates the presence of approximately 1000 genes that encode transporters, comprising approximately $4 \%$ of all genes (Clarke et al., 2002). The ATP-binding cassette and soluble carrier (SLC) transporter groups are the two major superfamilies of membrane transporter proteins that influence drug pharmacokinetics. SLC transporters, such as nucleoside, amino acid, and folate transporters, commonly increase cell chemosensitivity by mediating the cellular uptake of hydrophilic drugs (Candelaria et al., 2010). In humans, there are two types of structurally unrelated protein families that mediate nucleoside transport: the SLC28 family of cation-linked concentrative nucleoside transporters (CNTs) and the SLC29 family of energy-independent and equilibrative nucleoside transporters (ENTs) (Young, 2016). The human ENTs (hENTs) permit the bidirectional passage of pyrimidine and purine nucleosides, such as gemcitabine, capecitabine, and 5-fluorouracil (5FU), into cells (Yao et al., 2011). Azacytidine (AZA) and decitabine (DAC), DNA methyltransferase 1-inhibiting nucleoside analogues that restore gene expression silenced by hypermethylation, are also known to be taken up by hENT1 (Huang et al., 2004; Schneider-Stock et al., 2005; Ueda et al., 2015.

The human genome contains four SLC29 family genes (SLC29A1, SLC29A2, $S L C 29 A 3$, and $S L C 29 A 4$ ) that encode four hENT proteins (hENT1-4, respectively). hENT1 and hENT2 are present in most cell types and transport a broad range of pyrimidine and purine nucleosides. hENT1 recognizes gemcitabine as a substrate with relatively high affinity, whereas hENT2 may be considered a low-affinity transporter of this fluoropyrimidine (Mackey et al., 1999). A recent study showed that high hENT1 levels in tumor tissue correlate with a poor clinical response to $5 \mathrm{FU}$ in patients with colorectal cancer (CRC) (Phua et al., 2013).

First-line treatment of most CRCs involves chemotherapy with 5FU-based regimens (Gustavsson et al., 2015). In early-stage cases, surgery alone might be suitable for low-risk tumors. Nevertheless, nucleoside-derived chemotherapy might be useful in the treatment of tumors resistant to conventional chemotherapy (Yoshimatsu et al., 2003). Combinations of AZA or DAC in epigenetic therapies are under phase I studies in patients with advanced CRC. These features, along with the fact that CRCs show significant heterogeneity resulting in different molecular subtypes with different clinical behaviors, prompted us to study the expression profiles of SLC29A1 (hENT1) and SLC29A2 (hENT2) in different colon cancer cell lines.

Genetics and Molecular Research 16 (1): gmr16019549 


\section{MATERIAL AND METHODS}

\section{Cell culture}

Sixteen colon cancer cell lines, including 8 cell lines derived from metastatic sites (Colo201, Colo205, LoVo, SK-CO-1, SNU-C1, SW620, SW626, and T84) and 8 cell lines originating from primary sites (Caco2, Colo320, DLD1, HCT116, HT29, RKO, SW48, and SW480), were examined. All cell lines were acquired from the American Type Culture Collection (ATCC) and were grown as previously described.

\section{Total RNA extraction and reverse transcription}

Total RNA was isolated using an RNeasy Mini Kit (Qiagen, Valencia, CA, USA) according to the manufacturer's instructions. Briefly, cells were rinsed with PBS and scraped off in $750 \mu \mathrm{L}$ RNA Protect. The cells were pelleted by centrifugation, suspended in $350 \mu \mathrm{L}$ RLT buffer from the RNeasy kit, and homogenized using a Qiashredder spin column (Qiagen). The homogenized cell lysate effluent from the spin column was then processed as per the manufacturer's instructions of the RNeasy mini kit. The kit included a gDNA eliminator column, and an aqueous suspension of the total RNA was obtained. Concentrations and initial quality assessments of total RNA were determined by measuring the absorbance at 260, 230, and $280 \mathrm{~nm}$. The RNA quality of the samples was checked using Nano6000/Pico Bioanalyzer chips (Agilent 2100 Bioanalyzer, Santa Clara, CA, USA). One microgram of the total isolated cellular RNA was used for reverse transcription, which was performed as per the manufacturer's instructions of the High Capacity cDNA Reverse transcription kit (Applied Biosystems, Foster City, CA, USA).

\section{Quantitative real-time polymerase chain reaction analysis}

The target gene levels were determined by real-time reverse transcriptase polymerase chain reaction (RT-PCR). Each reaction contained $10.0 \mu \mathrm{L} 2 \mathrm{X}$ Power SYBR Green PCR Master Mix (Applied Biosystems), forward and reverse primers (2.5 pmol each), and $2 \mu \mathrm{L}$ cDNA template in a total reaction volume of $20 \mu \mathrm{L}$. To confirm the specificity of amplicons produced by PCR, we performed dissociation curve analysis. PCR was performed using an Applied Biosystems 7500 One-Step Plus Real-Time PCR System. Thermal cycling conditions were as follows: initial denaturation for $10 \mathrm{~min}$ at $95^{\circ} \mathrm{C}$, followed by 35 cycles of $30 \mathrm{~s}$ at $95^{\circ} \mathrm{C}$, annealing for $1 \mathrm{~min}$ at $60^{\circ} \mathrm{C}$, and $30 \mathrm{~s}$ at $72^{\circ} \mathrm{C}$. Samples were compared using the relative threshold cycles $(\mathrm{Ct})$ method. To normalize the input load of cDNA between samples, $G A P D H$ was used as an endogenous standard. The specific primers used are listed in Table 1. All experimental samples were analyzed in triplicates.

\section{Statistical analysis}

Results are reported as means \pm SE. Statistical analysis was performed using the SPSS software (version 18.0; SPSS, Chicago, IL, USA). The student $t$-test was used to test the significance between SLC29A1 and SLC29A2 levels in different cell lines. All tests were twosided, and a $\mathrm{P}$ value of less than 0.05 was considered significant.

Genetics and Molecular Research 16 (1): gmr16019549 


\section{Table 1. Primer sequences.}

\begin{tabular}{l|l|l}
\hline Gene & Forward & Reverse \\
\hline$S L C 29 A 1$ & 5'-GTGCCTTCGGCTACTTTATCAC-3' & 5'- GCTAATGAGGTCCAACTTGGTCT-3' \\
\hline SLC29A2 & 5'-TCAGTGCAGTCCTACAGGG-3' & 5'- GGCGTGATAAAGTACCCCAGG-3' \\
\hline GAPDH & 5'-ACAACTTTGGTATCGTGGAAGG-3' & 5'-GCCATCACGCCACAGTTTC-3' \\
\hline
\end{tabular}

\section{RESULTS}

\section{$S L C 29 A 1$ expression in 16 colon cancer cell lines}

SLC29A1 expression was detected in all 16 colon cancer cell lines (Figure 1). The mean expression value of all cell lines was 0.00217 . Notably, SLC29A1 was strongly expressed in two metastatic cell lines (Colo201 and Colo205) and 5 primary cell lines (Caco2, Colo320, HCT116, RKO, and SW48). Moreover, we compared the SLC29A1 expression levels in the primary colon cancer cell line SW480 with its lymph node metastatic counterpart SW620. The expression levels were lower in the metastatic cell line SW620 $(0.00079 \pm 0.00004)$ than in the parental primary tumor cell line SW480 $(0.00101 \pm 0.00005, \mathrm{P}=0.031)$. In general, the $S L C 29 A 1$ expression levels were lower in the 8 metastatic cell lines than in the 8 primary cell lines $(\mathrm{P}=0.003)$.

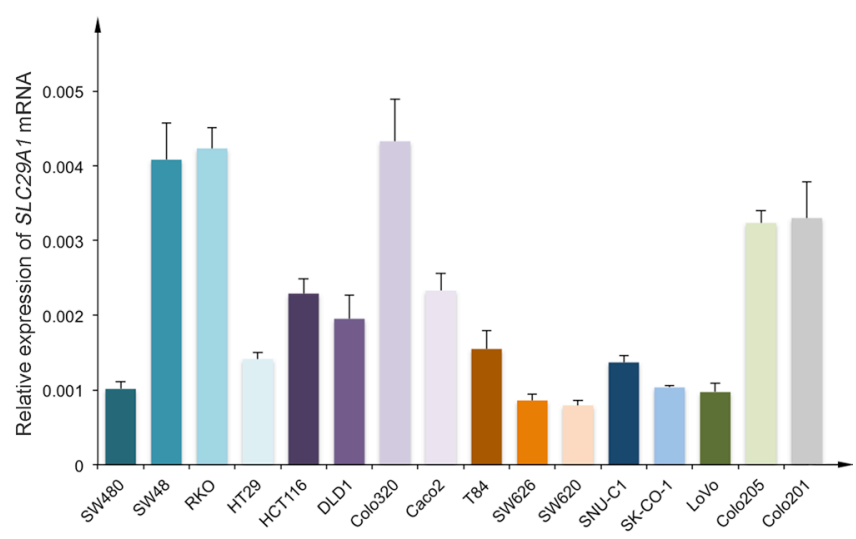

Figure 1. Differential expression of SLC29A1 in 16 colon cancer cell lines.

\section{$S L C 29 A 2$ expression in 16 colon cancer cell lines}

SLC29A2 expression was detected in all 16 colon cancer cell lines (Figure 2). The mean expression value of all cell lines was 0.00312 . Notably, SLC29A2 was strongly expressed in four metastatic cell lines (Colo205, LoVo, SK-CO-1, and T84) and 4 primary cell lines (Caco2, Colo320, HCT116, and HT29). The expression levels were significantly lower in the metastatic cell line SW620 (0.00136 \pm 0.00007$)$ than in the primary tumor cell line SW480 $(0.00206 \pm 0.00015, \mathrm{P}=0.014)$. In general, lower $S L C 29 A 2$ expression levels were observed in the 8 metastatic cell lines than in the 8 primary cell lines $(\mathrm{P}=0.043)$.

Genetics and Molecular Research 16 (1): gmr16019549 


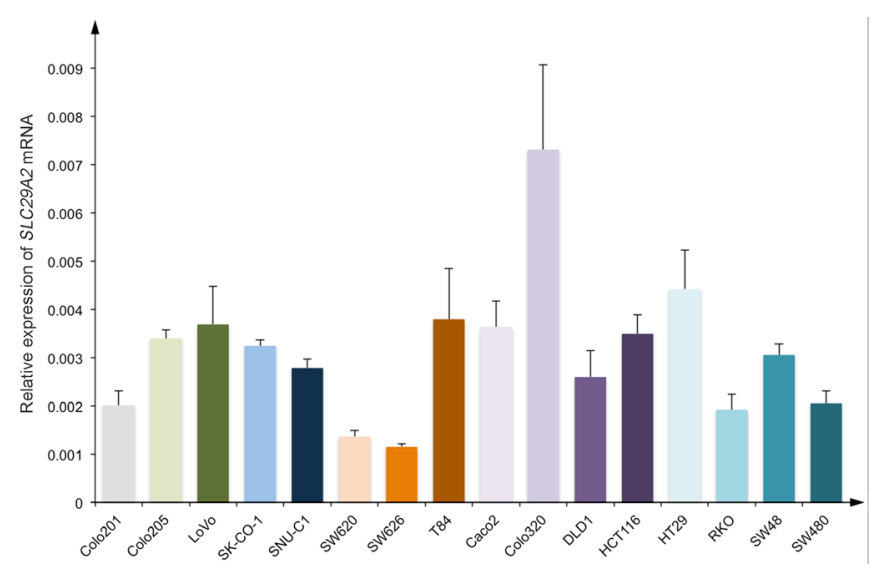

Figure 2. Differential expression of SLC29A2 in 16 colon cancer cell lines.

\section{DISCUSSION}

The RNA expression profiles of hENT1 and hENT2, encoded by SLC29A1 and $S L C 29 A 2$, in 16 colon cancer cell lines were analyzed. To the best of our knowledge, this study is the first to comprehensively profile the transcription levels of the main hENTs in a large number of colon cancer cell lines. SLC29A1 and SLC29A2 were differentially expressed in the 16 colon cancer cell lines. For the first time, we showed that colon cancer cell lines originating from metastatic sites had lower expression levels of both SLC29A1 and SLC29A2 than those observed in colon cancer cell lines originating from primary sites. In particular, the primary colon cancer cell line SW480 had significantly higher SLC29A1 and SLC29A2 expression levels than those observed in its lymph node metastatic counterpart SW620. These findings highlight the importance of evaluating the expression of transporters and differences in drug responses between primary and metastatic tumors in patients with metastatic CRC (mCRC).

In the adjuvant setting after complete resection, a molecular biomarker with predictive value could help in optimizing therapy by selecting an individualized chemotherapy regimen (Fisher et al., 2013). Since the majority of patients with mCRC ultimately die of the disease, predictive biomarkers are particularly important. Expression profiles of hENT have been assessed in a variety of cancers, including gastrointestinal, breast, biliary tract, pancreatic, bladder, and lung cancers, and shown to have predictive value for the efficacy of nucleoside-derived drugs (Achiwa et al., 2004; Giovannetti et al., 2006; Molina-Arcas et al., 2006; Oguri et al., 2007; Kameyama et al., 2011; Matsumura et al., 2011; Santini et al., 2008, 2011; Ormanns et al., 2014). Gemcitabine is a drug that requires active transport into the cell by hENT1 to function therapeutically (Mini et al., 2006). Several in vitro studies on various cancer cell lines have reported a direct correlation of hENT1 expression with gemcitabine sensitivity (Giovannetti et al., 2006; Mori et al., 2007). A clinical study showed that hENT1 was a promising response biomarker for patients with advanced pancreatic cancer treated with gemcitabine; high hENT1 expression correlated with longer survival (Spratlin et al., 2004). More importantly, a recent prospective study provided powerful evidence against the use of the adjuvant gemcitabine after resection for tumors with low hENT1 expression in patients with pancreatic cancer. 5FU-based regimens could be used instead and vice versa (Greenhalf et al., 2014).

Genetics and Molecular Research 16 (1): gmr16019549 
Although the efficacy of combined chemotherapy with gemcitabine and uracil-tegafur (UFT) as a salvage treatment in patients with mCRC was found to be unsatisfactory, a small proportion of patients showed prolonged tumor stabilization to gemcitabine and UFT (Lee et al., 2014). Several studies have also shown that a proportion of mCRC patients might benefit from combined chemotherapy with gemcitabine (Correale et al., 2014; Spindler et al., 2014). However, few studies have evaluated hENT expression profiles and their possible predictive value for chemotherapy in CRC. The expression of a panel of nucleoside transporters (hENT14 and hCNT1-2) in tumors samples of 7 CRC patients was investigated by real-time PCR prior to 5FU-based chemotherapy. High hENT1 levels in tumor tissue were shown to be correlated with poor clinical response to 5FU. Moreover, Caco-2 cell viability was measured following incubation with varying concentrations of $5 \mathrm{FU}$ and a specific hENT1 inhibitor. The inhibition of hENT1 resulted in an increase in 5FU efficacy in Caco-2 cells. Preliminary data suggested that hENT1 is a potential co-determinant of the clinical response to 5FU (Phua et al., 2013).

Using oligonucleotide arrays to analyze the gene expression profiles of transporters and channels in 60 human cancer cell lines, Huang et al. (2004) validated the positive correlation between SLC29A1 expression and AZA potency. The application of an hENT1 inhibitor, nitrobenzylmercaptopurine ribonucleoside, significantly reduced the potency of this drug, indicating that hENT1 plays a role in cellular uptake (Schneider-Stock et al., 2005; Ueda et al., 2015). Furthermore, hENT1 was identified as a crucial transporter in cellular AZA uptake in human leukemia cells (Hummel-Eisenbeiss et al., 2013). DAC is also transported by hENT1; this uptake was one of the key determinants of DAC activity in the colon cancer cell line HCT116 (Ueda et al., 2015). These results emphasize the important roles that multiple types of membrane transport molecules could play in the sensitivity and resistance to cytotoxic drugs.

In this study, sixteen colon cell lines, including 8 cell lines derived from metastatic sites (Colo201, Colo205, LoVo, SK-CO-1, SNU-C1, SW620, SW626, and T84) and 8 cell lines originating from primary sites (Caco2, Colo320, DLD1, HCT116, HT29, RKO, SW48, and SW480), were examined. We validated the ubiquitous and heterogeneous distribution of SLC29A1 and SLC29A2 in a large series of human colon cancer cell lines and demonstrated that $S L C 29 A 1$ was highly expressed in $25 \%$ of metastatic cell lines (Colo201 and Colo205) and 62.5\% of primary cell lines (Caco2, Colo320, HCT116, RKO, and SW48). However, SLC29A2 was strongly expressed in 50\% of metastatic cell lines (Colo205, LoVo, SK-CO-1, and T84) and 50\% of primary cell lines (Caco2, Colo320, HCT116, and HT29). In general, the 8 metastatic cell lines had lower SLC29A1 and $S L C 29 A 2$ expression levels than those observed in the 8 primary cell lines. The metastatic cell line SW620 had significantly lower expression levels of both genes than those observed in the parental primary tumor cell line SW480.

These findings indicate that, to some extent, most patients with mCRC may have low hENT1 expression, and treatment with nucleoside analogues, such as gemcitabine, AZA, and DAC, etc., may be inefficient. However, some patients still show high hENT1 expression and have a high probability of benefiting from these drug-based chemotherapies and developing resistance to 5FU-based chemotherapy. Further assessments of the role of hENTs in drug-based treatment of $\mathrm{mCRC}$ are required to elucidate the mechanisms of chemotherapy resistance.

\section{Conflicts of interest}

The other authors declare no conflict of interest.

Genetics and Molecular Research 16 (1): gmr16019549 


\section{ACKNOWLEDGMENTS}

Nita Ahuja received grant funding from Astex and has licensed biomarkers to Cepheid, Inc. She has served as a consultant for Ethicon, Inc. Research supported by National Institutes of Health grants \#CA140599 and \#CA127141 and the National Natural Science Foundation of China grants \#81000898 and \#81472289 and was sponsored by the Scientific Research Foundation for the Returned Overseas Chinese Scholars, State Education Ministry of China.

\section{REFERENCES}

Achiwa H, Oguri T, Sato S, Maeda H, et al. (2004). Determinants of sensitivity and resistance to gemcitabine: the roles of human equilibrative nucleoside transporter 1 and deoxycytidine kinase in non-small cell lung cancer. Cancer Sci. 95: 753-757. http://dx.doi.org/10.1111/j.1349-7006.2004.tb03257.x

Candelaria M, de la Cruz-Hernández E, Pérez-Cárdenas E, Trejo-Becerril C, et al. (2010). Pharmacogenetics and pharmacoepigenetics of gemcitabine. Med. Oncol. 27: 1133-1143. http://dx.doi.org/10.1007/s12032-009-9349-y

Clarke ML, Mackey JR, Baldwin SA, Young JD, et al. (2002). The role of membrane transporters in cellular resistance to anticancer nucleoside drugs. Cancer Treat. Res. 112: 27-47. http://dx.doi.org/10.1007/978-1-4615-1173-1_2

Correale P, Botta C, Rotundo MS, Guglielmo A, et al. (2014). Gemcitabine, oxaliplatin, levofolinate, 5-fluorouracil, granulocyte-macrophage colony-stimulating factor, and interleukin-2 (GOLFIG) versus FOLFOX chemotherapy in metastatic colorectal cancer patients: the GOLFIG-2 multicentric open-label randomized phase III trial. $J$. Immunother. 37: 26-35. http://dx.doi.org/10.1097/CJI.0000000000000004

Fisher SB, Patel SH, Bagci P, Kooby DA, et al. (2013). An analysis of human equilibrative nucleoside transporter-1, ribonucleoside reductase subunit M1, ribonucleoside reductase subunit M2, and excision repair cross-complementing gene-1 expression in patients with resected pancreas adenocarcinoma: implications for adjuvant treatment. Cancer 119: 445-453. http://dx.doi.org/10.1002/cncr.27619

Giovannetti E, Del Tacca M, Mey V, Funel N, et al. (2006). Transcription analysis of human equilibrative nucleoside transporter-1 predicts survival in pancreas cancer patients treated with gemcitabine. Cancer Res. 66: 3928-3935. http://dx.doi.org/10.1158/0008-5472.CAN-05-4203

Greenhalf W, Ghaneh P, Neoptolemos JP, Palmer DH, et al.; European Study Group for Pancreatic Cancer (2014). Pancreatic cancer hENT1 expression and survival from gemcitabine in patients from the ESPAC-3 trial. J. Natl. Cancer Inst. 106: djt347. http://dx.doi.org/10.1093/jnci/djt347

Gustavsson B, Carlsson G, Machover D, Petrelli N, et al. (2015). A review of the evolution of systemic chemotherapy in the management of colorectal cancer. Clin. Colorectal Cancer 14: 1-10. http://dx.doi.org/10.1016/j.clcc.2014.11.002

Huang Y, Anderle P, Bussey KJ, Barbacioru C, et al. (2004). Membrane transporters and channels: role of the transportome in cancer chemosensitivity and chemoresistance. Cancer Res. 64: 4294-4301. http://dx.doi.org/10.1158/0008-5472. CAN-03-3884

Hummel-Eisenbeiss J, Hascher A, Hals PA, Sandvold ML, et al. (2013). The role of human equilibrative nucleoside transporter 1 on the cellular transport of the DNA methyltransferase inhibitors 5-azacytidine and CP-4200 in human leukemia cells. Mol. Pharmacol. 84: 438-450. http://dx.doi.org/10.1124/mol.113.086801

Kameyama R, Yamamoto Y, Izuishi K, Sano T, et al. (2011). Correlation of 18F-FLT uptake with equilibrative nucleoside transporter-1 and thymidine kinase-1 expressions in gastrointestinal cancer. Nucl. Med. Commun. 32: 460-465. http:// dx.doi.org/10.1097/MNM.0b013e32834209b5

Lee KW, Kim YJ, Lee KH, Han SW, et al. (2014). Phase II trial of gemcitabine plus UFT as salvage treatment in oxaliplatin, irinotecan and fluoropyrimidine-refractory metastatic colorectal cancer. Cancer Chemother. Pharmacol. 74: 447-455. http://dx.doi.org/10.1007/s00280-014-2515-8

Mackey JR, Yao SY, Smith KM, Karpinski E, et al. (1999). Gemcitabine transport in xenopus oocytes expressing recombinant plasma membrane mammalian nucleoside transporters. J. Natl. Cancer Inst. 91: 1876-1881. http:// dx.doi.org/10.1093/jnci/91.21.1876

Matsumura N, Nakamura Y, Kohjimoto Y, Inagaki T, et al. (2011). The prognostic significance of human equilibrative nucleoside transporter 1 expression in patients with metastatic bladder cancer treated with gemcitabine-cisplatinbased combination chemotherapy. BJU Int. 108: E110-E116. http://dx.doi.org/10.1111/j.1464-410X.2010.09932.x

Mini E, Nobili S, Caciagli B, Landini I, et al. (2006). Cellular pharmacology of gemcitabine. Ann. Oncol. 17 (Suppl 5): v7-v12. http://dx.doi.org/10.1093/annonc/mdj941

Genetics and Molecular Research 16 (1): gmr16019549 
Molina-Arcas M, Moreno-Bueno G, Cano-Soldado P, Hernández-Vargas H, et al. (2006). Human equilibrative nucleoside transporter-1 (hENT1) is required for the transcriptomic response of the nucleoside-derived drug 5'-DFUR in breast cancer MCF7 cells. Biochem. Pharmacol. 72: 1646-1656. http://dx.doi.org/10.1016/j.bcp.2006.07.036

Mori R, Ishikawa T, Ichikawa Y, Taniguchi K, et al. (2007). Human equilibrative nucleoside transporter 1 is associated with the chemosensitivity of gemcitabine in human pancreatic adenocarcinoma and biliary tract carcinoma cells. Oncol. Rep. 17: 1201-1205.

Oguri T, Achiwa H, Muramatsu H, Ozasa H, et al. (2007). The absence of human equilibrative nucleoside transporter 1 expression predicts nonresponse to gemcitabine-containing chemotherapy in non-small cell lung cancer. Cancer Lett. 256: 112-119. http://dx.doi.org/10.1016/j.canlet.2007.06.012

Ormanns S, Heinemann V, Raponi M, Isaacson J, et al. (2014). Human equilibrative nucleoside transporter 1 is not predictive for gemcitabine efficacy in advanced pancreatic cancer: translational results from the AIO-PK0104 phase III study with the clone SP120 rabbit antibody. Eur. J. Cancer 50: 1891-1899. http://dx.doi.org/10.1016/j. ejca.2014.04.023

Phua LC, Mal M, Koh PK, Cheah PY, et al. (2013). Investigating the role of nucleoside transporters in the resistance of colorectal cancer to 5-fluorouracil therapy. Cancer Chemother. Pharmacol. 71: 817-823. http://dx.doi.org/10.1007/ $\underline{\mathrm{s} 00280-012-2054-0}$

Santini D, Perrone G, Vincenzi B, Lai R, et al. (2008). Human equilibrative nucleoside transporter 1 (hENT1) protein is associated with short survival in resected ampullary cancer. Ann. Oncol. 19: 724-728. http://dx.doi.org/10.1093/ annonc/mdm576

Santini D, Schiavon G, Vincenzi B, Cass CE, et al. (2011). Human equilibrative nucleoside transporter 1 (hENT1) levels predict response to gemcitabine in patients with biliary tract cancer (BTC). Curr. Cancer Drug Targets 11: 123-129. http://dx.doi.org/10.2174/156800911793743600

Schneider-Stock R, Diab-Assef M, Rohrbeck A, Foltzer-Jourdainne C, et al. (2005). 5-Aza-cytidine is a potent inhibitor of DNA methyltransferase 3a and induces apoptosis in HCT-116 colon cancer cells via Gadd45- and p53-dependent mechanisms. J. Pharmacol. Exp. Ther. 312: 525-536. http://dx.doi.org/10.1124/jpet.104.074195

Spindler KL, Pallisgaard N, Andersen RF, Ploen J, et al. (2014). Gemcitabine and capecitabine for heavily pre-treated metastatic colorectal cancer patients-a phase II and translational research study. Anticancer Res. 34: 845-850.

Spratlin J, Sangha R, Glubrecht D, Dabbagh L, et al. (2004). The absence of human equilibrative nucleoside transporter 1 is associated with reduced survival in patients with gemcitabine-treated pancreas adenocarcinoma. Clin. Cancer Res. 10: 6956-6961. http://dx.doi.org/10.1158/1078-0432.CCR-04-0224

Ueda K, Hosokawa M and Iwakawa S (2015). Cellular uptake of decitabine by equilibrative nucleoside transporters in HCT116 Cells. Biol. Pharm. Bull. 38: 1113-1119. http://dx.doi.org/10.1248/bpb.b14-00622

Yao SY, Ng AM, Cass CE, Baldwin SA, et al. (2011). Nucleobase transport by human equilibrative nucleoside transporter 1 (hENT1). J. Biol. Chem. 286: 32552-32562. http://dx.doi.org/10.1074/jbc.M111.236117

Yoshimatsu K, Kato H, Ishibashi K, Hashimoto M, et al. (2003). Second-line chemotherapy with low-dose CPT-11 and cisplatin for colorectal cancer resistant to 5-FU-based chemotherapy. Cancer Chemother. Pharmacol. 52: 465-468. http://dx.doi.org/10.1007/s00280-003-0686-9

Young JD (2016). The SLC28 (CNT) and SLC29 (ENT) nucleoside transporter families: a 30-year collaborative odyssey. Biochem. Soc. Trans. 44: 869-876. http://dx.doi.org/10.1042/BST20160038

Genetics and Molecular Research 16 (1): gmr16019549 